\title{
Numerical Tests with Gauss-Type Nested Implicit Runge-Kutta Formulas ${ }^{\star}$
}

\author{
Gennady Yu. Kulikov and Sergey K. Shindin
}

School of Computational and Applied Mathematics, University of the Witwatersrand, Private Bag 3, Wits 2050, Johannesburg, South Africa

gkulikov@cam.wits.ac.za, sshindin@cam.wits.ac.za

\begin{abstract}
In this paper we conduct a detailed numerical analysis of the Gauss-type Nested Implicit Runge-Kutta formulas of order 4, introduced by Kulikov and Shindin in 4. These methods possess many important practical properties such as high order, good stability, symmetry and so on. They are also conjugate to a symplectic method of order 6 at least. All of these make them efficient for solving many nonstiff and stiff ordinary differential equations (including Hamiltonian and reversible systems). On the other hand, Nested Implicit Runge-Kutta formulas have only explicit internal stages, in the sense that they are easily reduced to a single equation of the same dimension as the source problem. This means that such Runge-Kutta schemes admit a cheap implementation in practice. Here, we check the above-mentioned properties numerically. Different strategies of error estimation are also examined with the purpose of finding an effective one.
\end{abstract}

\section{Introduction}

In this paper we deal with numerical solution of ordinary differential equations (ODE's) of the form

$$
x^{\prime}(t)=g(t, x(t)), \quad t \in\left[t_{0}, t_{\text {end }}\right], \quad x\left(t_{0}\right)=x^{0}
$$

where $x(t) \in \mathbb{R}^{n}$ and $g: D \subset \mathbb{R}^{n+1} \rightarrow \mathbb{R}^{n}$ is a sufficiently smooth function. Problem (11) is assumed to have a unique solution $x(t)$ on the interval $\left[t_{0}, t_{\text {end }}\right]$.

An l-stage Nested Implicit Runge-Kutta (NIRK) formula applied to ODE (1) reads

$$
\begin{aligned}
& x_{k j}^{2}=a_{j 1}^{2} x_{k}+a_{j 2}^{2} x_{k+1}+\tau_{k}\left(d_{j 1}^{2} g\left(t_{k}, x_{k}\right)+d_{j 2}^{2} g\left(t_{k+1}, x_{k+1}\right)\right), \\
& x_{k j}^{i}=a_{j 1}^{i} x_{k}+a_{j 2}^{i} x_{k+1}+\tau_{k}\left(d_{j 1}^{i} g\left(t_{k}, x_{k}\right)+d_{j 2}^{i} g\left(t_{k+1}, x_{k+1}\right)\right) \\
& \quad+\tau_{k} \sum_{m=1}^{i-1} d_{j, m+2}^{i} g\left(t_{k m}^{i-1}, x_{k m}^{i-1}\right), \quad i=3,4, \ldots, l, \quad j=1,2, \ldots, i,
\end{aligned}
$$

\footnotetext{
* This work was supported in part by the National Research Foundation of South Africa under grant No. FA2004033000016.
} 


$$
x_{k+1}=x_{k}+\tau_{k} \sum_{i=1}^{l} b_{i} g\left(t_{k i}^{l}, x_{k i}^{l}\right) .
$$

where $x_{0}=x^{0}, t_{k j}^{i}=t_{k}+\tau_{k} c_{j}^{i}$ and $\tau_{k}$ is a step size. It is also required that $a_{j 1}^{i}+a_{j 2}^{i}=1$ and $c_{j}^{i}=a_{j 2}^{i}+\sum_{m=1}^{i+1} d_{j m}^{i}$. We stress that method (2) is an RK formula and its Butcher tableau is given by

\begin{tabular}{c|ccccccc}
0 & 0 & 0 & 0 & $\ldots$ & 0 & 0 & 0 \\
$c^{2}$ & $d_{1}^{2}$ & 0 & 0 & $\ldots$ & 0 & $a^{2} b^{T}$ & $d_{2}^{2}$ \\
$c^{3}$ & $d_{1}^{3}$ & $D^{3}$ & 0 & $\ldots$ & 0 & $a^{3} b^{T}$ & $d_{2}^{3}$ \\
$c^{4}$ & $d_{1}^{4}$ & 0 & $D^{4}$ & $\ldots$ & 0 & $a^{4} b^{T}$ & $d_{2}^{4}$ \\
$\vdots$ & $\vdots$ & $\vdots$ & $\vdots$ & $\ddots$ & $\vdots$ & $\vdots$ & $\vdots$ \\
$c^{l}$ & $d_{1}^{l}$ & 0 & 0 & $\ldots$ & $D^{l}$ & $a^{l} b^{T}$ & $d_{2}^{l}$ \\
1 & 0 & 0 & 0 & $\ldots$ & 0 & $b^{T}$ & 0 \\
\hline & 0 & 0 & 0 & $\cdots$ & 0 & $b^{T}$ & 0
\end{tabular}

where

$$
\begin{gathered}
D^{i}=\left(\begin{array}{ccc}
d_{12}^{i} & \cdots & d_{1, i+1}^{i} \\
\vdots & \ddots & \vdots \\
d_{i 2}^{i} & \cdots & d_{i, i+1}^{i}
\end{array}\right), \quad d_{1}^{i}=\left(\begin{array}{c}
d_{11}^{i} \\
\vdots \\
d_{i 1}^{i}
\end{array}\right), \quad d_{2}^{i}=\left(\begin{array}{c}
d_{12}^{i} \\
\vdots \\
d_{i 2}^{i}
\end{array}\right), \\
a^{i}=\left(\begin{array}{c}
a_{12}^{i} \\
\vdots \\
a_{i 2}^{i}
\end{array}\right), \quad c^{i}=\left(\begin{array}{c}
c_{1}^{i} \\
\vdots \\
c_{i}^{i}
\end{array}\right), \quad b=\left(\begin{array}{c}
b_{1} \\
\vdots \\
b_{l}
\end{array}\right) .
\end{gathered}
$$

It is quite clear that NIRK method (2) is easily reduced to a single equation of dimension $n$. This is of great advantage in practice. We refer the reader to [4] and [6] for more information about the reason to design NIRK formulas. Our intention here is to examine numerically the Gauss-type NIRK methods of order 4 introduced in the cited papers. Especially, we are interested to test adaptive formulas of this type to find a proper computational technique for practical use.

Finally, we want to point out that the first research on the topic under discussion was done by van Bokhoven 1, who introduced cheap RK methods termed Implicit Endpoint Quadrature (IEQ) formulas. It is obvious that NIRK methods are a particular case of IEQ formulas. Nevertheless, van Bokhoven made some mistakes in his paper and failed to find good practical methods, in the sense of the properties mentioned above (see 6] for explanation). Thus, we concentrate here on the NIRK methods designed by Kulikov and Shindin.

\section{Adaptive Gauss-Type NIRK Methods}

The Gauss-type NIRK methods of orders 2, 4 and 6 have been developed and investigated theoretically in [4] and 6]. So we intend to discuss different error 
estimations which are suitable for the NIRK methods in Section 2. We do our analysis for the methods of order 4 . However, the results obtained can be extended to the Gauss-type NIRK formulas of order 6. Our research covers both Embedded Methods Approach (EMA) and Richardson Extrapolation Technique (RET) as well. We will see below that methods (2) admit one more idea for error estimation, termed Embedded Stages Approach (ESA).

We emphasize that van Bokhoven 1] failed to construct RK schemes with built-in error estimation which are effective to treat stiff ODE's (see 6] for details). Therefore we apply EMA to solve this problem for Gauss-type NIRK methods of order 4 . In other words, we deal with embedded RK formulas of the form

\begin{tabular}{c|cccc}
0 & 0 & 0 & 0 & 0 \\
$c_{1}^{2}$ & $\frac{6\left(c_{1}^{2}+\theta\right)-5}{12}$ & $\frac{1-\theta}{2}$ & $\frac{1-\theta}{2}$ & $\frac{6\left(c_{1}^{2}+\theta\right)-7}{12}$ \\
$1-c_{1}^{2}$ & $\frac{7-6\left(c_{1}^{2}+\theta\right)}{12}$ & $\frac{\theta}{2}$ & $\frac{\theta}{2}$ & $\frac{5-6\left(c_{1}^{2}+\theta\right)}{12}$ \\
\hline 1 & 0 & $\frac{1}{2}$ & $\frac{1}{2}$ & 0 \\
\hline \multirow{2}{*}{} & $\frac{1}{2}$ & $\frac{1}{2}$ & $\frac{1}{2}$ & 0 \\
\hline & 0 & $-\frac{1}{2}$ & $-\frac{1}{2}$ & $\frac{1}{2}$
\end{tabular}

where $c_{1}^{2}=(3-\sqrt{3}) / 6$ and $\theta$ is a real free parameter. We recall that NIRK method (4) is of stage order 3 when $\theta=1 / 2+2 \sqrt{3} / 9$ and of stage order 2 otherwise (see [4]). In this paper we are going to examine numerically the following five different error estimations introduced in [6]:

Embedded Methods Error Estimation (EMEE) is taken to be

$$
l e_{k+1}=\frac{\tau_{k}}{2}\left(g\left(t_{k}, x_{k}\right)-g\left(t_{k 1}^{2}, x_{k 1}^{2}\right)-g\left(t_{k 2}^{2}, x_{k 2}^{2}\right)+g\left(t_{k+1}, x_{k+1}\right)\right) .
$$

Estimate (5) is cheap and of order 3. It is based on Trapezoidal Rule. Unfortunately, one of the RK schemes in the embedded pair (4) is not $A$-stable. Hence, EMEE can be inefficient for some stiff ODE's. That is why we offer an improved error estimation based on Shampine's idea in the above-cited paper.

Modified Embedded Methods Error Estimation (MEMEE) uses the formula

$$
Q_{1}\left(\tau_{k} J\right) \tilde{l e}_{k+1}=l e_{k+1}
$$

where $Q_{1}(z)=(1-z / 4)^{3}$ and $J$ means the Jacobi matrix evaluated at the point $\left(t_{k}, x_{k}\right)$. The last formula implies that the improved error estimate is obtained by solving linear system (6). We stress that MEMEE is not expensive in practice because it means three solutions of linear systems with the coefficient matrix $I-\tau_{k} J / 4$. Anyway, the latter matrix is computed and decomposed to advance a step of method (4) (see [4] and [5] for more detail). Eventually, MEMEE is suitable for integration of stiff problems (see 6]). 
One more idea of error estimation discovered in the above-mentioned paper and referred to as Embedded Stages Error Estimation (ESEE) uses the fact that calculation of stage values is explicit and, hence, very cheap in NIRK methods. We know that method (4) is of stage order 3 when $\theta=1 / 2+2 \sqrt{3} / 9$. It is of stage order 2 for other $\theta$ 's. Therefore we choose another $\hat{\theta} \neq 1 / 2+2 \sqrt{3} / 9$ to calculate the stage values of the different order. When taking the difference we come to the error estimate

$$
\hat{l e}_{k+1}=\frac{\tau_{k}}{8}\left(g\left(t_{k}, x_{k}\right)-g\left(t_{k 1}^{2}, x_{k 1}^{2}\right)-g\left(t_{k 2}^{2}, x_{k 2}^{2}\right)+g\left(t_{k+1}, x_{k+1}\right)\right) .
$$

ESEE is of order 3 and used in a stepsize selection algorithm in the usual way. Again, error estimate (7) can be inefficient to treat stiff ODE's.

Hopefully, a modification is possible to make ESEE suitable for stiff problems. For them, it is recommended to apply Modified Embedded Stages Error Estimation (MESEE) presented by the formula

$$
Q_{2}(\tau J) \overline{l e}_{k+1}=\frac{\tau_{k}}{8}\left(g\left(t_{k}, x_{k}\right)-g\left(t_{k 1}^{2}, x_{k 1}^{2}\right)-g\left(t_{k 2}^{2}, x_{k 2}^{2}\right)+g\left(t_{k+1}, x_{k+1}\right)\right)
$$

where $Q_{2}(z)=1-z / 4$. It is not difficult to check that error estimate (8) is limited for any step size. It is also cheap because of the reasons given above. We point out that the last formula is superior to error estimation (6) in terms of CPU time since only one extra solution is implemented in MESEE.

For the sake of completeness, we include Richardson Extrapolation Error Estimation (REEE) applied to the Gauss-type NIRK method of order 4 when $\theta=1 / 2+2 \sqrt{3} / 9$ in our testing below. Details of REEE can be found in [3], for example. We stress that the NIRK method of order 4 and with REEE showed sufficiently good numerical results when applied to the two-dimensional Brusselator with diffusion and the periodic boundary conditions. We compared it with the usual Gauss method of order 4 which uses the same error estimation and stepsize selection strategy (see [4).

\section{Test Problems}

In the experiments below we want to check how our NIRK methods perform for problems of different sorts. We cover Hamiltonian systems and the usual nonstiff and stiff ODE's. We also include some ODE's with known exact solutions in order to check the quality of numerical solutions obtained. This allows us to exhibit practical properties of the adaptive NIRK methods introduced in 4] and 6]. We are interested to find the methods which compute good numerical solutions for the minimum CPU time. To implement our plan we take the following test problems:

The Kepler problem is given by

$$
x_{1}^{\prime \prime}(t)=\frac{-x_{1}(t)}{\left(x_{1}^{2}(t)+x_{2}^{2}(t)\right)^{3 / 2}}, \quad x_{2}^{\prime \prime}(t)=\frac{-x_{2}(t)}{\left(x_{1}^{2}(t)+x_{2}^{2}(t)\right)^{3 / 2}}
$$


where $x_{1}(0)=1-e, x_{1}^{\prime}(0)=0, x_{2}(0)=0, x_{2}^{\prime}(0)=\sqrt{1+e} / \sqrt{1-e}$ and $e=$ 0.2. Problem (9) has two first integrals: the total energy $H\left(x^{\prime}(t), x(t)\right)$ and the angular momentum $L\left(x^{\prime}(t), x(t)\right)$ where $x(t)=\left(x_{1}(t), x_{2}(t)\right)$. Its exact solution is also well-known. Thus, the Kepler problem is considered to be a good example of Hamiltonian systems and is often used to test numerical methods suitable for such sort of problems (see, for instance, [2]).

The simple problem is presented by

$$
\begin{gathered}
x_{1}^{\prime}(t)=2 t x_{2}^{1 / 5}(t) x_{4}(t), \quad x_{2}^{\prime}(t)=10 t \exp \left(5\left(x_{3}(t)-1\right)\right) x_{4}(t), \\
x_{3}^{\prime}(t)=2 t x_{4}(t), \quad x_{4}^{\prime}(t)=-2 t \ln \left(x_{1}(t)\right), \quad t \in[0,5]
\end{gathered}
$$

where $x(0)=(1,1,1,1)^{T}$. It possesses the exact solution

$x_{1}(t)=\exp \left(\sin t^{2}\right), \quad x_{2}(t)=\exp \left(5 \sin t^{2}\right), \quad x_{3}(t)=\sin t^{2}+1, \quad x_{4}(t)=\cos t^{2}$,

which will be used to check the quality of numerical solutions obtained in our experiments.

The restricted three body problem is

$$
\begin{gathered}
x_{1}^{\prime \prime}(t)=x_{1}(t)+2 x_{2}^{\prime}(t)-\mu_{1} \frac{x_{1}(t)+\mu_{2}}{y_{1}(t)}-\mu_{2} \frac{x_{1}(t)-\mu_{1}}{y_{2}(t)}, \\
x_{2}^{\prime \prime}(t)=x_{2}(t)-2 x_{1}^{\prime}(t)-\mu_{1} \frac{x_{2}(t)}{y_{1}(t)}-\mu_{2} \frac{x_{2}(t)}{y_{2}(t)}, \\
y_{1}(t)=\left(\left(x_{1}(t)+\mu_{2}\right)^{2}+x_{2}^{2}(t)\right)^{3 / 2}, y_{2}(t)=\left(\left(x_{1}(t)-\mu_{1}\right)^{2}+x_{2}^{2}(t)\right)^{3 / 2}
\end{gathered}
$$

where $t \in[0, T], T=17.065216560157962558891, \mu_{1}=1-\mu_{2}$ and $\mu_{2}=$ 0.012277471 . The initial values of problem (11) are: $x_{1}(0)=0.994, x_{1}^{\prime}(0)=0$, $x_{2}(0)=0, x_{2}^{\prime}(0)=-2.00158510637908252240$. Problem (11) has no analytic solution, but it is still useful to gain experience concerning the quality of numerical solutions because its solution-path is periodic. We can monitor the error at the end point $T$ to verify the quality.

A pattern of stiff ODE's is presented by Van der Pol's equation

$$
x_{1}^{\prime}(t)=x_{2}(t), \quad x_{2}^{\prime}(x)=\mu^{2}\left(\left(1-x_{1}^{2}(t)\right) x_{2}(t)-x_{1}(t)\right), \quad t \in[0,2]
$$

where $x(0)=(2,0)^{T}$ and $\mu=1000$. Problem (12) is considered to be very stiff.

\section{Numerical Experiments}

We start this section with numerical experiments on the Kepler problem. We stress that the standard stepsize selection is not suitable for integration of Hamiltonian and reversible problems because it ruins nice properties of specially designed numerical methods (see Section VIII in [2]). So we just apply the fixedstepsize version of method (4) when $\theta=1 / 2+2 \sqrt{3} / 9$ to see how it works for long time integration of problem (9). 

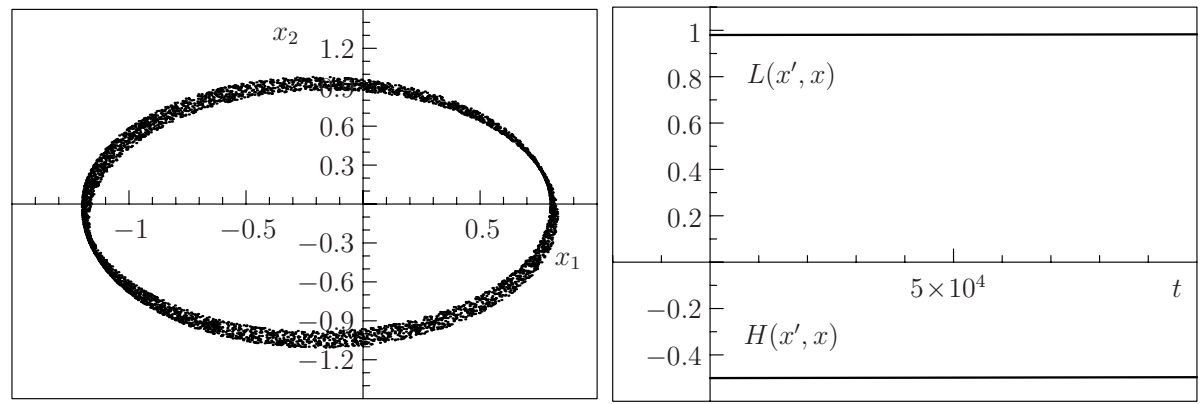

Fig. 1. The numerical solution of the Kepler problem (the left-hand graph) and its first integrals (the right-hand graph) computed by the fixed-stepsize Gauss-type NIRK method of order 4

Table 1. The global errors of the adaptive Gauss-type NIRK method of order 4 with different error estimation techniques applied to the simple problem

\begin{tabular}{r|ccccc}
\hline \multirow{2}{*}{$\begin{array}{c}\text { Error } \\
\text { Tolerance }\end{array}$} & EMEE & ESEE & MEMEE & MESEE & REEE \\
\hline $10^{-1}$ & $3.139 \cdot 10^{+1}$ & $1.199 \cdot 10^{+2}$ & $3.646 \cdot 10^{+1}$ & $1.479 \cdot 10^{+2}$ & $2.534 \cdot 10^{+2}$ \\
$5 \cdot 10^{-2}$ & $1.876 \cdot 10^{+1}$ & $6.355 \cdot 10^{+1}$ & $1.409 \cdot 10^{+1}$ & $1.557 \cdot 10^{+2}$ & $1.750 \cdot 10^{+2}$ \\
$10^{-2}$ & $1.766 \cdot 10^{+0}$ & $1.779 \cdot 10^{+1}$ & $1.575 \cdot 10^{+0}$ & $1.720 \cdot 10^{+1}$ & $2.450 \cdot 10^{+1}$ \\
$5 \cdot 10^{-3}$ & $7.694 \cdot 10^{-1}$ & $4.566 \cdot 10^{+0}$ & $6.969 \cdot 10^{-1}$ & $4.605 \cdot 10^{+0}$ & $1.183 \cdot 10^{+1}$ \\
$10^{-3}$ & $7.740 \cdot 10^{-2}$ & $5.502 \cdot 10^{-1}$ & $7.568 \cdot 10^{-2}$ & $5.624 \cdot 10^{-1}$ & $3.207 \cdot 10^{+0}$ \\
$5 \cdot 10^{-4}$ & $2.501 \cdot 10^{-2}$ & $2.055 \cdot 10^{-1}$ & $2.582 \cdot 10^{-2}$ & $2.096 \cdot 10^{-1}$ & $1.311 \cdot 10^{+0}$ \\
$10^{-4}$ & $2.051 \cdot 10^{-3}$ & $1.731 \cdot 10^{-2}$ & $1.839 \cdot 10^{-3}$ & $1.706 \cdot 10^{-2}$ & $1.547 \cdot 10^{-1}$ \\
$5 \cdot 10^{-5}$ & $6.609 \cdot 10^{-4}$ & $5.961 \cdot 10^{-3}$ & $6.650 \cdot 10^{-4}$ & $5.944 \cdot 10^{-3}$ & $6.716 \cdot 10^{-2}$ \\
$10^{-5}$ & $7.155 \cdot 10^{-5}$ & $4.976 \cdot 10^{-4}$ & $6.533 \cdot 10^{-5}$ & $5.005 \cdot 10^{-4}$ & $1.385 \cdot 10^{-2}$ \\
\hline
\end{tabular}

Figure 1 shows the behaviour of the numerical solution and the first integrals calculated by our NIRK method with the step size $\tau_{k}=0.1$ on the interval $\left[0,10^{+5}\right]$. We observe that this result corresponds well to the exact solution and the first integrals of the Kepler problem (see [2, p. 9]). Thus, method (4) is suitable for the numerical integration of Hamiltonian and reversible problems in practice. We recall that the method under discussion is conjugate to a symplectic method of order 6 at least (see [6]).

Now we come to tests with the adaptive Gauss-type NIRK methods. For that, we perform a number of numerical integrations of problems (10) and (11) by the method (4) when $\theta=1 / 2+2 \sqrt{3} / 9$ and with the error estimation strategies mentioned in Section 2. To implement the Gauss-type NIRK method, we apply a modified Newton iteration with nontrivial predictor, as explained in [4] or [5]. We perform two iteration steps per each grid point and display the global 
Table 2. CPU time (in sec.) of the adaptive Gauss-type NIRK method of order 4 with different error estimation techniques applied to the simple problem

\begin{tabular}{r|ccccc}
\hline Error & \multicolumn{5}{|c}{ Error Estimation Technique } \\
Tolerance & EMEE & ESEE & MEMEE & MESEE & REEE \\
\hline $10^{-1}$ & $0.375 \cdot 10^{+0}$ & $0.235 \cdot 10^{+0}$ & $0.328 \cdot 10^{+0}$ & $0.141 \cdot 10^{+0}$ & $0.328 \cdot 10^{+0}$ \\
$5 \cdot 10^{-2}$ & $0.328 \cdot 10^{+0}$ & $0.266 \cdot 10^{+0}$ & $0.391 \cdot 10^{+0}$ & $0.235 \cdot 10^{+0}$ & $0.391 \cdot 10^{+0}$ \\
$10^{-2}$ & $0.547 \cdot 10^{+0}$ & $0.422 \cdot 10^{+0}$ & $0.531 \cdot 10^{+0}$ & $0.391 \cdot 10^{+0}$ & $0.453 \cdot 10^{+0}$ \\
$5 \cdot 10^{-3}$ & $0.579 \cdot 10^{+0}$ & $0.437 \cdot 10^{+0}$ & $0.657 \cdot 10^{+0}$ & $0.422 \cdot 10^{+0}$ & $0.469 \cdot 10^{+0}$ \\
$10^{-3}$ & $0.938 \cdot 10^{+0}$ & $0.625 \cdot 10^{+0}$ & $1.047 \cdot 10^{+0}$ & $0.609 \cdot 10^{+0}$ & $0.500 \cdot 10^{+0}$ \\
$5 \cdot 10^{-4}$ & $1.204 \cdot 10^{+0}$ & $0.781 \cdot 10^{+0}$ & $1.219 \cdot 10^{+0}$ & $0.828 \cdot 10^{+0}$ & $0.547 \cdot 10^{+0}$ \\
$10^{-4}$ & $1.844 \cdot 10^{+0}$ & $1.328 \cdot 10^{+0}$ & $1.875 \cdot 10^{+0}$ & $1.343 \cdot 10^{+0}$ & $0.672 \cdot 10^{+0}$ \\
$5 \cdot 10^{-5}$ & $2.391 \cdot 10^{+0}$ & $1.500 \cdot 10^{+0}$ & $2.422 \cdot 10^{+0}$ & $1.687 \cdot 10^{+0}$ & $0.719 \cdot 10^{+0}$ \\
$10^{-5}$ & $3.984 \cdot 10^{+0}$ & $2.672 \cdot 10^{+0}$ & $3.985 \cdot 10^{+0}$ & $2.656 \cdot 10^{+0}$ & $1.031 \cdot 10^{+0}$ \\
\hline
\end{tabular}

Table 3. The errors evaluated at the point $T$ for the adaptive Gauss-type NIRK method of order 4 with different error estimation techniques applied to the restricted three body problem

\begin{tabular}{r|ccccc}
\hline Error & \multicolumn{5}{|c}{ Error Estimation Technique } \\
Tolerance & EMEE & ESEE & MEMEE & MESEE & REEE \\
\hline $10^{-1}$ & $1.988 \cdot 10^{+0}$ & $8.816 \cdot 10^{+1}$ & $1.347 \cdot 10^{+0}$ & $3.474 \cdot 10^{+0}$ & $1.832 \cdot 10^{+0}$ \\
$5 \cdot 10^{-2}$ & $1.900 \cdot 10^{+0}$ & $2.163 \cdot 10^{+0}$ & $1.813 \cdot 10^{+0}$ & $1.823 \cdot 10^{+0}$ & $1.915 \cdot 10^{+0}$ \\
$10^{-2}$ & $9.913 \cdot 10^{-1}$ & $1.945 \cdot 10^{+0}$ & $8.998 \cdot 10^{-1}$ & $1.898 \cdot 10^{+0}$ & $1.435 \cdot 10^{+0}$ \\
$5 \cdot 10^{-3}$ & $4.124 \cdot 10^{-1}$ & $1.580 \cdot 10^{+0}$ & $6.277 \cdot 10^{-1}$ & $1.536 \cdot 10^{+0}$ & $1.330 \cdot 10^{+0}$ \\
$10^{-3}$ & $1.485 \cdot 10^{-1}$ & $4.496 \cdot 10^{-1}$ & $1.695 \cdot 10^{-1}$ & $4.768 \cdot 10^{-1}$ & $8.324 \cdot 10^{-1}$ \\
$5 \cdot 10^{-4}$ & $7.058 \cdot 10^{-2}$ & $2.721 \cdot 10^{-1}$ & $8.218 \cdot 10^{-2}$ & $2.955 \cdot 10^{-1}$ & $1.821 \cdot 10^{-1}$ \\
$10^{-4}$ & $1.092 \cdot 10^{-2}$ & $5.593 \cdot 10^{-2}$ & $1.180 \cdot 10^{-2}$ & $5.825 \cdot 10^{-2}$ & $3.470 \cdot 10^{-2}$ \\
$5 \cdot 10^{-5}$ & $4.336 \cdot 10^{-3}$ & $2.471 \cdot 10^{-2}$ & $4.610 \cdot 10^{-3}$ & $2.557 \cdot 10^{-2}$ & $4.986 \cdot 10^{-3}$ \\
$10^{-5}$ & $5.306 \cdot 10^{-4}$ & $3.224 \cdot 10^{-3}$ & $5.530 \cdot 10^{-4}$ & $3.282 \cdot 10^{-3}$ & $1.925 \cdot 10^{-3}$ \\
\hline
\end{tabular}

errors calculated by the exact solutions of these test problems in the sup-norm in Tables 1 and 3. The CPU time of these integrations is shown in Tables 2 and 4. respectively. The CPU time for numerical solutions of Van der Pol's equation is presented in Table 5.

Tables 1 5 show clearly that ESEE and MESEE produce numerical solutions of good quality for less CPU time. Certainly, REEE works faster when applied to problems of small size because this estimate is of order 5 . The remaining estimates are of order 3. On the other hand, REEE is very expensive for largescale problems (see Table 1 in [6]). In conclusion, we consider that the error estimation based on ESA is quite promising and deserves further study. We intend to develop such error estimation techniques for the Gauss-type NIRK method of order 6 . In future it is also planned to supply our NIRK schemes with a facility to provide Automatic Global Error Control. 
Table 4. CPU time (in sec.) of the adaptive Gauss-type NIRK method of order 4 with different error estimation techniques applied to the restricted three body problem

\begin{tabular}{r|ccccc}
\hline Error & \multicolumn{5}{|c}{ Error Estimation Technique } \\
Tolerance & EMEE & ESEE & MEMEE & MESEE & REEE \\
\hline $10^{-1}$ & $0.156 \cdot 10^{+0}$ & $0.062 \cdot 10^{+0}$ & $0.094 \cdot 10^{+0}$ & $0.047 \cdot 10^{+0}$ & $0.187 \cdot 10^{+0}$ \\
$5 \cdot 10^{-2}$ & $0.140 \cdot 10^{+0}$ & $0.125 \cdot 10^{+0}$ & $0.125 \cdot 10^{+0}$ & $0.078 \cdot 10^{+0}$ & $0.235 \cdot 10^{+0}$ \\
$10^{-2}$ & $0.204 \cdot 10^{+0}$ & $0.125 \cdot 10^{+0}$ & $0.203 \cdot 10^{+0}$ & $0.109 \cdot 10^{+0}$ & $0.281 \cdot 10^{+0}$ \\
$5 \cdot 10^{-3}$ & $0.266 \cdot 10^{+0}$ & $0.156 \cdot 10^{+0}$ & $0.219 \cdot 10^{+0}$ & $0.156 \cdot 10^{+0}$ & $0.281 \cdot 10^{+0}$ \\
$10^{-3}$ & $0.359 \cdot 10^{+0}$ & $0.235 \cdot 10^{+0}$ & $0.391 \cdot 10^{+0}$ & $0.250 \cdot 10^{+0}$ & $0.360 \cdot 10^{+0}$ \\
$5 \cdot 10^{-4}$ & $0.468 \cdot 10^{+0}$ & $0.312 \cdot 10^{+0}$ & $0.453 \cdot 10^{+0}$ & $0.328 \cdot 10^{+0}$ & $0.375 \cdot 10^{+0}$ \\
$10^{-4}$ & $0.672 \cdot 10^{+0}$ & $0.484 \cdot 10^{+0}$ & $0.657 \cdot 10^{+0}$ & $0.515 \cdot 10^{+0}$ & $0.438 \cdot 10^{+0}$ \\
$5 \cdot 10^{-5}$ & $0.797 \cdot 10^{+0}$ & $0.531 \cdot 10^{+0}$ & $0.813 \cdot 10^{+0}$ & $0.625 \cdot 10^{+0}$ & $0.532 \cdot 10^{+0}$ \\
$10^{-5}$ & $1.250 \cdot 10^{+0}$ & $0.906 \cdot 10^{+0}$ & $1.266 \cdot 10^{+0}$ & $0.891 \cdot 10^{+0}$ & $0.703 \cdot 10^{+0}$ \\
\hline
\end{tabular}

Table 5. CPU time (in sec.) of the adaptive Gauss-type NIRK method of order 4 with different error estimation techniques applied to Van der Pol's equation

\begin{tabular}{r|ccccc}
\hline \multirow{2}{*}{ Error } & \multicolumn{5}{|c}{ Error Estimation Technique } \\
Tolerance & EMEE & ESEE & MEMEE & MESEE & REEE \\
\hline $10^{-1}$ & $3.219 \cdot 10^{+0}$ & $2.406 \cdot 10^{+0}$ & $3.593 \cdot 10^{+0}$ & $1.734 \cdot 10^{+0}$ & $1.796 \cdot 10^{+0}$ \\
$5 \cdot 10^{-2}$ & $3.703 \cdot 10^{+0}$ & $2.859 \cdot 10^{+0}$ & $2.687 \cdot 10^{+0}$ & $2.047 \cdot 10^{+0}$ & $1.547 \cdot 10^{+0}$ \\
$10^{-2}$ & $5.516 \cdot 10^{+0}$ & $3.984 \cdot 10^{+0}$ & $6.234 \cdot 10^{+0}$ & $2.937 \cdot 10^{+0}$ & $1.641 \cdot 10^{+0}$ \\
$5 \cdot 10^{-3}$ & $6.531 \cdot 10^{+0}$ & $4.500 \cdot 10^{+0}$ & $5.250 \cdot 10^{+0}$ & $3.516 \cdot 10^{+0}$ & $2.625 \cdot 10^{+0}$ \\
$10^{-3}$ & $1.053 \cdot 10^{+1}$ & $6.922 \cdot 10^{+0}$ & $8.563 \cdot 10^{+0}$ & $5.562 \cdot 10^{+0}$ & $2.828 \cdot 10^{+0}$ \\
$5 \cdot 10^{-4}$ & $1.276 \cdot 10^{+1}$ & $8.578 \cdot 10^{+0}$ & $1.053 \cdot 10^{+1}$ & $6.985 \cdot 10^{+0}$ & $2.937 \cdot 10^{+0}$ \\
$10^{-4}$ & $2.090 \cdot 10^{+1}$ & $1.379 \cdot 10^{+1}$ & $1.806 \cdot 10^{+1}$ & $1.168 \cdot 10^{+1}$ & $3.797 \cdot 10^{+0}$ \\
$5 \cdot 10^{-5}$ & $2.646 \cdot 10^{+1}$ & $1.736 \cdot 10^{+1}$ & $2.285 \cdot 10^{+1}$ & $1.479 \cdot 10^{+1}$ & $4.422 \cdot 10^{+0}$ \\
$10^{-5}$ & $4.325 \cdot 10^{+1}$ & $2.782 \cdot 10^{+1}$ & $3.731 \cdot 10^{+1}$ & $2.457 \cdot 10^{+1}$ & $6.000 \cdot 10^{+0}$ \\
\hline
\end{tabular}

\section{References}

1. Van Bokhoven, W.M.G.: Efficient higher order implicit one-step methods for integration of stiff differential equations. BIT. 20 (1980) 34-43

2. Hairer, E., Lubich, C., Wanner, G.: Geometric numerical integration: Structure preserving algorithms for ordinary differential equations, Springer-Verlag, Berlin, 2002

3. Hairer, E., Nørsett, S.P., Wanner, G.: Solving ordinary differential equations I: Nonstiff problems. Springer-Verlag, Berlin, 1993

4. Kulikov, G.Yu., Shindin, S.K.: On a family of cheap symmetric one-step methods of order four. In: Vassil N. Alexandrov et al (eds.): Computational Science ICCS 2006. 6th International Conference, Reading, UK, May 28-31, 2006. Proceedings, Part I. Lecture Notes in Computer Science. 3991 (2006) 781-785

5. Kulikov, G.Yu., Merkulov, A.I., Shindin, S.K.: Asymptotic error estimate for general Newton-type methods and its application to differential equations. Russ. J. Numer. Anal. Math. Model. 22 (2007) (to appear)

6. Kulikov, G.Yu., Shindin, S.K.: Adaptive nested implicit Runge-Kutta formulas of the Gauss type (in preparation) 